\title{
Exploiting Vertical Diversity in Vehicular Channel Environments
}

\author{
Sangho Oh, Sanjit Kaul, Marco Gruteser \\ Electrical \& Computer Engineering, Rutgers University, 94 Brett Rd, Piscataway NJ 08854 \\ Email: \{sangho, sanjit, gruteser\}@winlab.rutgers.edu
}

\begin{abstract}
Antenna diversity is a well-known technique used to improve the quality and reliability of a wireless link. In vehicular networks, a different approach to antenna diversity is needed due to their unique channel characteristics. However, this issue has not been actively researched, especially for the positioning of antennas. In this paper, we highlight the benefit of vertical diversity over traditional horizontal diversity techniques in vehicular network environments. Through experiments using IEEE 802.11a radios in the $5.2 \mathrm{GHz}$ band, we first show the difference of attenuation patterns from various antenna positions installed in a vehicle, then we show the benefit of vertical diversity by quantifying the diversity gains and combined error rates. This finding has implications for the future position of antenna installation in vehicles.
\end{abstract}

Index Terms - antenna diversity, vertical diversity, vehicular network channel, ad-hoc network, broadcasting, two-ray ground model, height diversity

\section{INTRODUCTION}

Vehicular Ad-Hoc Network (VANET) has been studied over a decade as an important component in Intelligent Transportation Systems (ITS) that aim to provide comforts and safety to drivers and passengers on the road. Motivated by this, many projects have been launched related to the communications in VANET environments, e.g., Wireless Access in Vehicular Environment (WAVE) [1], PEeVENT [2], FleetNet [3], and Network on Wheels (NoW) [4]. They have actively developed various protocols that are specifically designed for vehicular network conditions.

Among many research topics on VANET, reliable communications in Vehicle-to-Vehicle (V2V) and Vehicle-toInfrastructure (V2I) is one of the fundamental issues, which is very closely related to the wireless channel characteristics. Although mobile communication channels have been thoroughly investigated over several decades in cellular network systems, VANET channel characteristics have only recently been studied from the perspective of mobile Ad-hoc networks.

VANET has unique channel characteristics. VANET communications experience location dependent outage probabilities due to a strong interference from the ground reflections [5] even when a link has a Line-Of-Sight (LOS) connection. Interference from a strong out-of-phase ground reflected signal generates a number of deep fades at specific locations outage spots - between the transmitter and the receiver. The receiving vehicles at the outage spots will experience temporal disconnections. For road safety applications, such as collision warning or pothole alarms, the outage spots can lead to critical

\section{situations.}

In such cases, antenna diversity techniques exploiting spatial diversity can be used to improve the reliability in communications. However, although the position of antenna for diversity greatly affects the performance of communication devices in VANET, this issues has not been thoroughly investigated considering the VANET specific channel characteristic.

In this paper, we show using experimentally collected data from a US highway that vertical antenna diversity better copes with strong interference from ground reflections than horizontal antenna diversity. We first show the channel characteristics caused by a strong ground reflections through a controlled parking lot experiment, then move on to the road experiments on a highway to verity how vertical diversify improves the reliability of communications. By quantifying the gains from vertical diversity over horizontal diversity for various transmitter-receiver distances, we demonstrate the benefits of vertical diversity.

\section{RELATED WORK}

Andrisano builds a propagation model for V2V channels considering the interference from ground reflections and multipath fading in the spectrum of millimeter wave $(60 \mathrm{GHz}) . \mathrm{He}$ finds the outage probability [6], [7] between two vehicles on a road. Ebine and Yamada measured the gains from a vertically spaced diversity antenna mounted on a vehicle [8]. However the system is a cellular system utilizing tall base station antennas, and the spatial diversity gain is achieved from the diversity in radiation patterns. Verdone proved the suitability of a multi-hop network approach for Road Transport Information (RTI) applications in $60 \mathrm{GHz}$ band, which is based on R-ALOHA protocol [5]. He assumed two-ray Rician fading channels with vertical diversity in V2V links. He showed how vertical diversity helps the connectivity in multi-hop protocols in VANET through simulations, but has not conducted an experimental validation. Kaul et al., conducted experiments on the diversity gains in vehicular network environments by comparing the azimuthal patterns of each of antennas that are horizontally and vertically displaced on top of the roof of a vehicle [9]. They discuss traditional diversity gains from horizontally displaced antennas over small scale fading in VANET environments. 


\section{System MODEL AND APPROACH}

The wireless channel in mobile networks can be modeled by combining a large-scale path-loss model and a small-scale fading model. Large-scale path-loss is usually modeled by a simple log-distance model with a path-loss exponent that varies depending on the terrain. The path-loss of Line-ofSight (LOS) conditions in VANET can be represented by a well known two-path deterministic model in (1) at TransmitterReceiver (Tx-Rx) distance $d$ [10]. $P_{r 0}$ is the log-distance path loss component with a path-loss exponent of $\eta$, and $h_{t}, h_{r}$ are the height of antennas of Tx and Rx respectively. $\pi$ is the phase difference between the two signals due to the reflection, and $r$ is the reflection coefficient that is close to 1 for smoothly paved roads [11].

$$
P_{r}=P_{r 0}\left[1+r^{2}+2 r \cos \varphi\right], \quad \varphi=\frac{4 \pi h_{t} h_{r}}{\lambda d}
$$

\section{A. Vertical Diversity}

In vehicular networks, the strong out-of-phased signal which is reflected from the paved road generates a number of outage spots between a transmitter and a receiver. Figure 1 shows the path loss for $\mathrm{V} 2 \mathrm{~V}$ cases. The outage spots are generated between $20-200 \mathrm{~m}$ assuming $-87 \mathrm{dBm}$ sensitivity of $802.11 \mathrm{a}$ radios. However, by exploiting the spatial diversity from the second antenna, which is vertically displaced $0.4 \mathrm{~m}$ from the other antenna, the probability of outage can be significantly reduced. On the contrary, the horizontal diversity using the Antenna-3, which is horizontally displaced $1.0 \mathrm{~m}$ from the other antenna, does not prevent the outages even with a displacement of $1.0 \mathrm{~m}$ from the other antenna. This is due to the fact that the horizontal antenna displacement only affects $\varphi$ marginally. Its diversity gain over ground reflection is minimal. This can be explained from the difference in paths between the two signals, $\Delta=\sqrt{\left(h_{t}+h_{r}\right)^{2}+d^{2}}-\sqrt{\left(h_{t}-h_{r}\right)^{2}+d^{2}}$, is predominantly affected by the height difference between the antennas.

In addition to the gains over ground reflection effects, vertically displacement can also draw gains from spatial diversity for multi-path fading as long as the displacement of two antennas are larger than half of the wavelength of the signal [10].

\section{EXPERIMENT SETUP}

This experiment aims to characterize the path-loss in VANET communication scenarios, and to characterize the benefit of vertical antenna diversity. Our measurements are based on 802.11a systems, which share lots of similar parts in the radio specifications with 802.11p that is designed for DSRC (Dedicated Short Range Communications) [12]. The system used for this experiment is shown in Fig. 2(a). We use ORBIT nodes [13] connected to GPS modules for location and Tx-Rx distance tracking.

By monitoring the number of packets received from the receiver, we quantify the gains from link level antenna diversity performance using Multi-Radio Packet Selection (MRPS). The

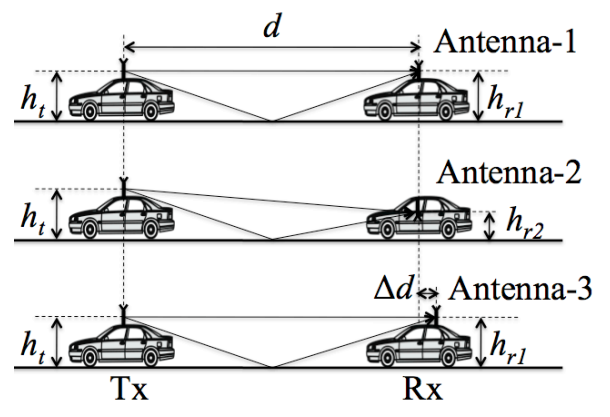

(a) Antenna positions and the signal propagation paths

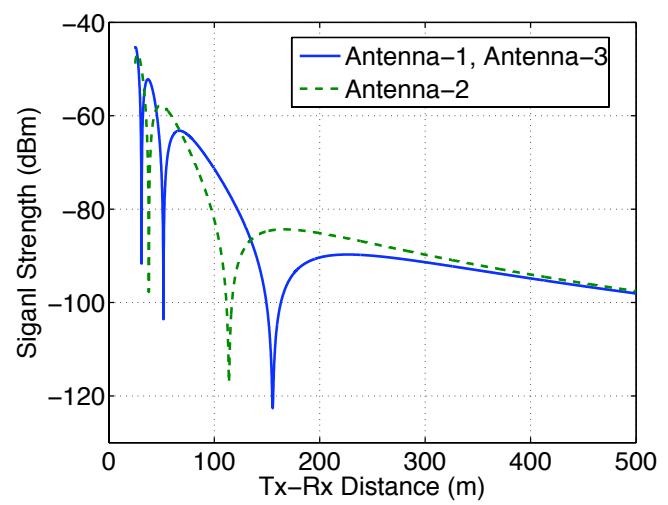

(b) Vertical antennal diversity (Antenna-2) vs. Horizontal antenna diversity (Antenna-3), Tx power $=100 \mathrm{mw}, h_{t}=1.5 \mathrm{~m}$, $h_{r 1}=1.6 \mathrm{~m}, h_{r 2}=1.2 \mathrm{~m}, \Delta d=1 \mathrm{~m}$, Frequency $=5.18 \mathrm{GHz}$, $r=1$

Fig. 1. Two-ray ground model

transmitter sends 1000 802.11 ICMP packets per second, and the receivers on each antenna count the number of packets received in the monitor mode. The placement of antennas is shown in Fig. 2(b). We installed six antennas, one primary antenna on the center of the roof (CE), four other roof antennas on FD (Front Driver), FP (Front Passenger), RD (Rear Driver), RP (Rear Passenger), and one antenna inside the car (IN) under the rear-view mirror.

IN is vertically displaced about $0.4 \mathrm{~m}$ from $\mathrm{CE}$, and the other four antennas are horizontally displaced from CE. Placing IN under the rear-view mirror, we considered following factors:

- The antenna has omni-directional sensitivity through the windshield glasses

- The position achieves good communication with a vehicle in the front, which may transmit collision warning messages

- Highest position as possible to prevent blockage by obstacles on the road

- The position enables communication with internal wireless devices inside of the vehicle with minimum interference to neighbor vehicles

Quantifying the performance of vertical diversity, we use following metrics:

1) Diversity gain: The number of packets corrected by MRPS using the second antenna over the number of packets 


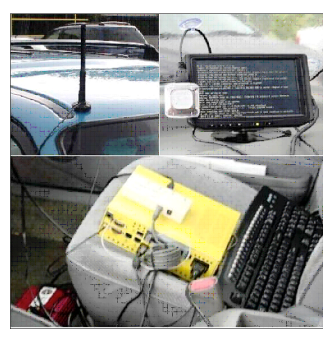

(a) Hardware Setup

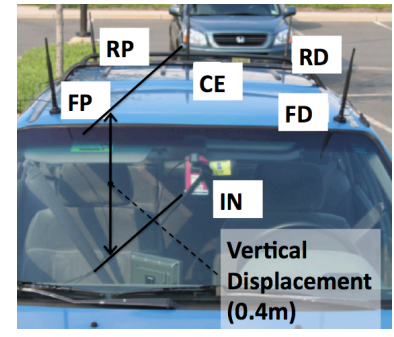

(b) Placement of Antennas

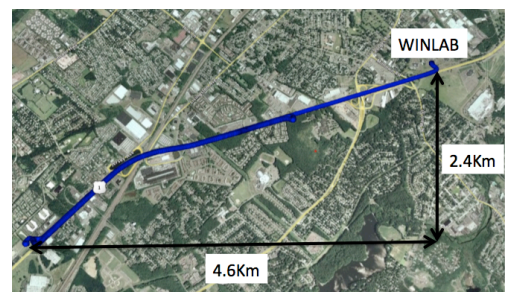

(c) Drive Route: US-1 Highway

Fig. 2. Experiments Configuration

transmitted from the transmitter

2) Combined error rate: The number of errors after MRPS over the number of packets transmitted from the transmitter.

The details of the experiment setup is summarized in Table. I, with the driving route shown in Fig. 2(c).

\section{A. Experiment Scenarios}

To experimentally quantify the gains from vertical diversity, first, we measure the received signal strength in an empty parking lot to verify the effects from ground reflections, which gives the motivation for vertical antenna diversity. A transmitter vehicle is parked at a fixed location, and the receiver vehicle moves toward and away from the transmitter. We measure how antenna positions affect the Received Signal Strength Indicator (RSSI) at the receiver network card. Motivated by this, we move on to the on-road experiments based on following two scenarios.

1) Two cars chasing scenario: A transmitter vehicle runs ahead and a receiver vehicle follows the transmitter. The vehicles run at average $50 \mathrm{mph}$ speed, and the antennas maintain LOS most of the time. The duration of the experiment is $1000 \mathrm{~s}$.

2) Two cars head on scenario: Both the transmitter and the receiver vehicle drive in opposite direction, and pass each other three times. The communication is only enabled when they are within a communicable range. The average speed of the vehicles is $50 \mathrm{mph}$ when they cross each other.

\section{REsults}

\section{A. Parking lot measurement}

In the parking lot experiment, we compare the effects from antenna position on the attenuation of the signal under the strong ground reflection environment. We compare signal strengths from each of antenna to understand the difference between horizontal diversity and vertical diversity in Fig. 3.
TABLE I

DEFAUlT EXPERIMENTAL PARAMETERS USED

\begin{tabular}{|l|c|}
\hline Parameter & Value \\
\hline \hline Wireless Network Card Modem & Atheros 5212 chipset \\
\hline Device Driver & MadWifi \\
\hline MAC and PHY protocol & $802.11 \mathrm{a}$ \\
\hline Frequency & $5.18 \mathrm{GHz}$ \\
\hline Transmit Power & $40 \mathrm{~mW}$ \\
\hline Antenna Type & folded dipole \\
\hline Antenna Gain & $3 \mathrm{dBi}$ \\
\hline PHY Data Rate & $6 \mathrm{Mbps}$ \\
\hline ICMP Payload size & 56 bytes \\
\hline Transmission Frequency & 1000 packets per second \\
\hline
\end{tabular}

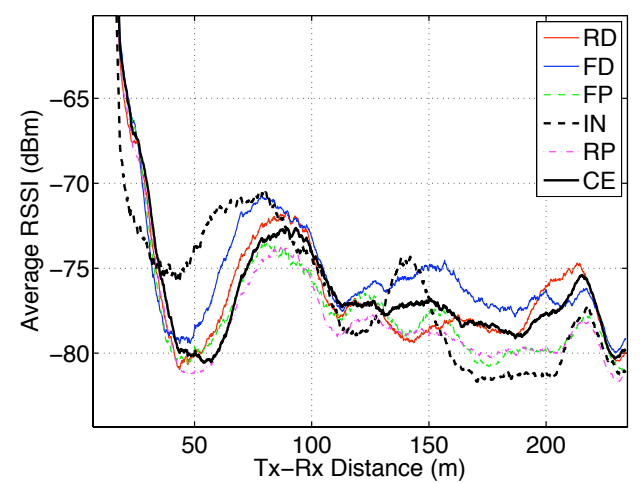

Fig. 3. Two-ray ground path loss in the empty parking lot (Livingston campus parking lot in Rutgers university, NJ)

Due to the ground reflection effects, the received signal suffers from non-uniform attenuation. However, the dip and rise of the signal has different patterns depending on the antenna positions. IN, which is vertically displaced from other four antennas, shows significantly different patterns from the other antennas, hence intuitively motivates the best diversity gains.

\section{B. Two cars chasing scenario}

In this scenario, we compare diversity gains and combined error rates for each of antenna positions. The result is averaged over the whole experiment period of $1000 \mathrm{~s}$, and then is analyzed over distance in $10 \mathrm{~m}$ granularity. As shown in Fig. 4, the diversity gain is maximized by vertically displaced antenna (IN) rather than by horizontally displace antennas installed on the roof of the test vehicle. Figure 4(a) and 4(b) shows per antenna performance, and Fig. 4(c) and 4(d) compare the performance of vertical diversity with the average performance of all horizontal antennas. The result indicates vertical diversity significantly reduce the effects from ground reflections that cause non-uniform reception rate over distance.

Although FP and RP show very good performance at $150-180 \mathrm{~m}$ ranges, horizontal diversity only partially solves the problem because its performance is not consistent over distance. Another problem of horizontal diversity is the difficulty in finding the best combination of horizontal antennas as it depends on the environmental situations and the shape of the vehicles. 


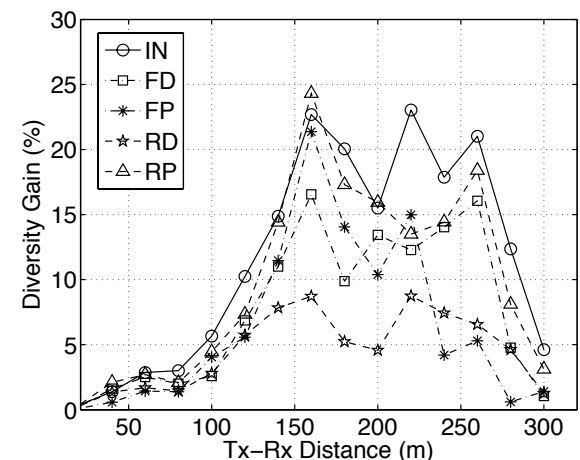

(a) Vertical diversity gains from the inside antenna (IN) and horizontal diversity gains from roof antennas (FD, FP, $\mathrm{RD}, \mathrm{RP})$

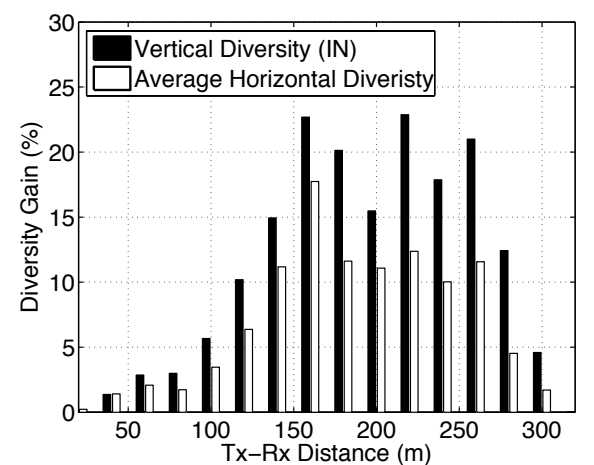

(c) Vertical diversity gains from the inside antenna (IN) and the averaged horizontal diversity gains over distance

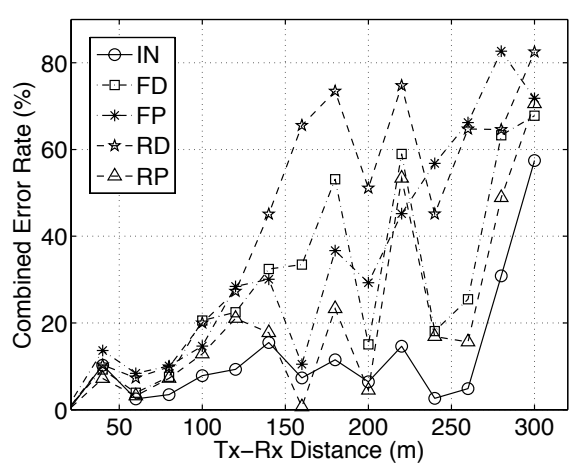

(b) Error rate after MRPS of the received packets

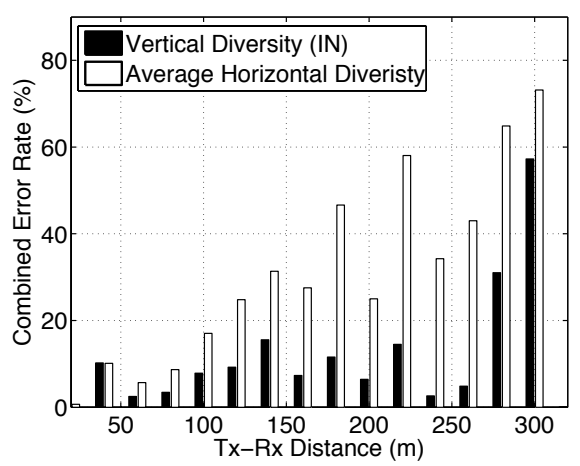

(d) Error rate after MRPS of the received packets over the distance

Fig. 4. Vertical Diversity Gain: Two cars chasing scenario (US-1, NJ)

In Fig. 5, we show the path-loss curve in the highway scenario using a Boxplot with $95 \%$ percentile. The resemblance of dips and rise of the signal to parking lot model indicates the ground reflection model still holds for highway scenarios, where the vertical diversity can get most of gains. However, the objects reflecting or blocking the signals in highway induce small-scale signal fluctuations, which differentiates the channel environment from that of controlled parking lot.

\section{Two cars head on scenario}

In this scenario, we measured how vertical diversity helps the early reception of collision warning signals through the experiments using two vehicles head on, running in opposite directions. We averaged the data collected from the three times the vehicles pass each other. Figure 6 shows the combined reception rate from diversity - one secondary antenna and the primary antenna - which is shown with the reception rate of the single primary antennas. The diversity gain from front antennas is small as their reception patterns are not significantly different from that of CE. However, IN achieves significant diversity gains in $180-250 \mathrm{~m}$ range. From these results, we verify that vertical diversity extends the coverage up to $50 \mathrm{~m}$ for head on running scenario while horizontal antenna diversity does not show significant improvement in packet receptions. We skipped the result for RP which shows similar performance with RD.

\section{CONCLUSION}

This is the first experimental research that has characterized the benefits of vertical diversity in VANET channel conditions. In traditional mobile networks, the gains from spatial diversity are primarily used to cope with small scale fading in non-LOS multi-path environments. However, in vehicular networks, where antennas are positioned at similar height and a strong interference from ground reflections exists, vertical antenna diversity performs significantly better than horizontal antenna diversity. While our experiments covered a limited set of traffic conditions, they showed that:

- In vehicular networks, location dependent communication outages possibly occur due to strong ground reflections

- Vertical diversity prevents possible connection loss due to the outage spots caused by horizontal reflections from the road

In urban multi-path channel environments, where horizontal and vertical reflections are mixed, the location dependent outage spots may not exist, however, vertical diversity can also exploit gains from spatial diversity like horizontal diversity against the small-scale fadings. 


\section{Center (CE)}
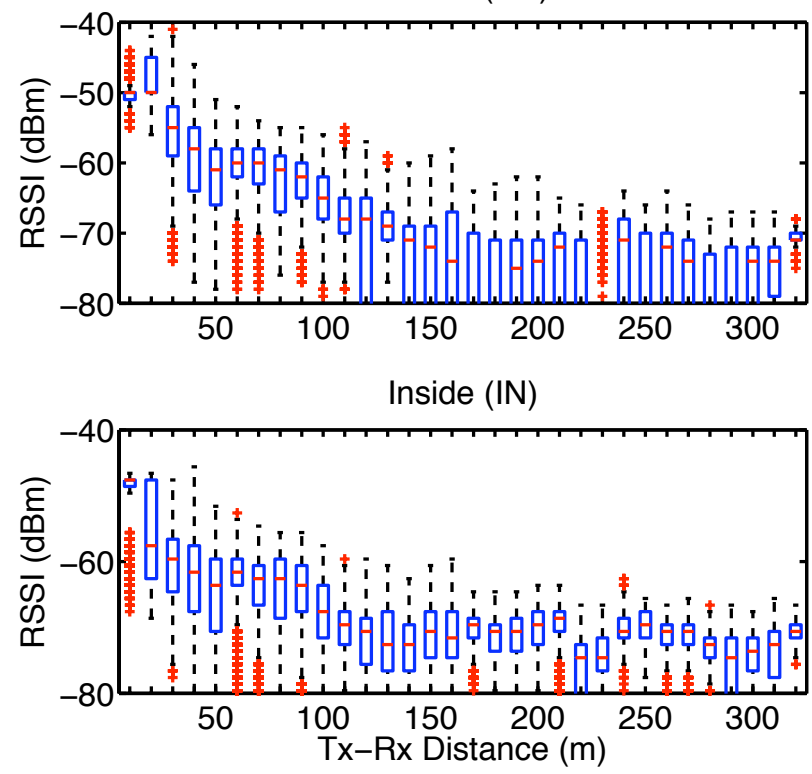

Fig. 5. Path-loss in the highway US-1, NJ
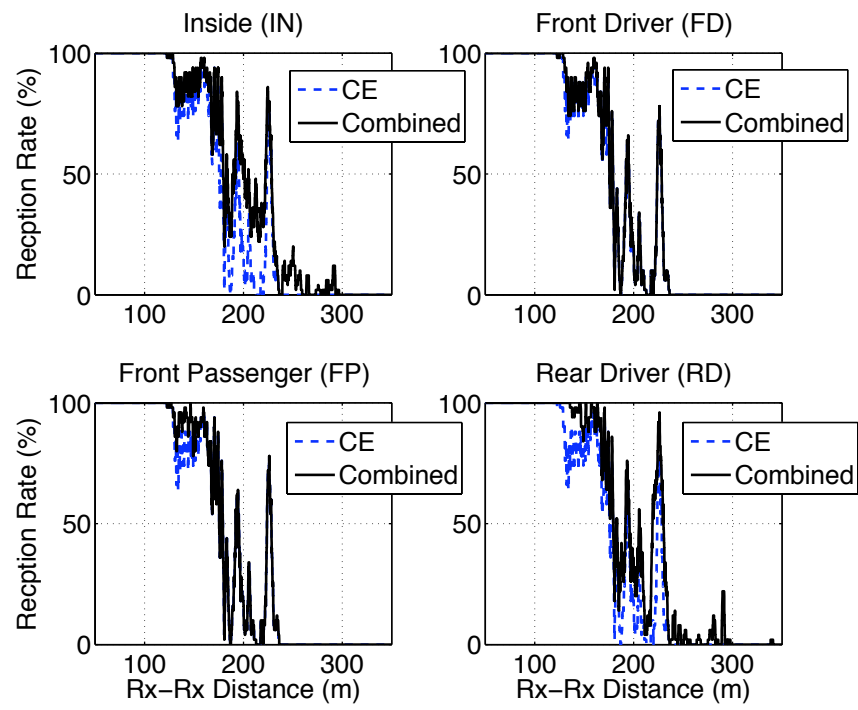

Fig. 6. Extension of the coverage from vertical diversity: Two cars head on running scenario (US-1, NJ)

\section{REFERENCES}

[1] IEEE trial-use standard for wireless access in vehicular environments (WAVE)multi-channel operation, IEEE std 1609.3. http://www. standards.its.dot.gov, 2007.

[2] PReVENT: Preventive and active safety; 6th framework program integrated project. http://www.prevent-ip.org.

[3] Project: FleetNet - internet on the road. http://www.et2.tu-harburg.de/ fleetnet/english/about.html.

[4] Network on Wheels Project. http://www.network-on-wheels.de/vision. html, 2007.

[5] Roberto Verdone. Multihop R-ALOHA for inter-vehicle communications at millimeter waves. IEEE Transactions on Vehicular Technology, 46:992-1005, Nov 1997.
[6] O. Andrisano, M. Chiani, V. Tralli, and R. Verdone. Impact of cochannel interference on vehicle-to-vehicle communications at millimeter waves. volume 2, pages 924-928, Nov 1992.

[7] O. Andrisano, M. Chiani, M. Frullone, C. Moss, and V. Tralli. Propagation effects and countermeasures analysis in vehicle-to-vehicle communication at millimeter waves. volume 1, pages 312-316, May 1992.

[8] Y. Ebine and Y. Yamada. A vehicular-mounted vertical space diversity antenna for a land mobile radio. IEEE Transactions on Vehicular Technology, 40(2):420-425, May 1991.

[9] S. Kaul, K. Ramachandran, P. Shankar, S. Oh, M. Gruteser, I. Seskar, and T. Nadeem. Effect of antenna placement and diversity on vehicular network communications. In Sensor, Mesh and Ad Hoc Communications and Network (SECON), pages 112-121, 2007.

[10] Theodore S. Rappaport. Wireless Communications: Principles and Practice (2nd Edition). Prentice Hall PTR, Dec 2001.

[11] John S. Seybold. Introduction to RF propagation. Technology \& Engineering, 2005

[12] Dedicated short range communications (DSRC). http://www.standards its.dot.gov.

[13] D. Raychaudhuri, I. Seskar, M. Ott, S. Ganu, K. Ramachandran, H. Kremo, R. Siracusa, H. Liu, and M. Singh. Overview of the ORBIT radio grid testbed for evaluation of next-generation wireless network protocols. In IEEE WCNC, March 2005. 